\title{
Lack of epidermal growth factor receptor $(E G F R)$-activating mutations in triple-negative breast cancer in China
}

\author{
Wen-Ming Cao ${ }^{1,2}$, Yun Gao ${ }^{2,3}$ and Xiao-Jia Wang ${ }^{1,2^{*}}$ \\ See related research by Teng et al., http://www.breast-cancer-research.com/content/13/2/R35
}

We read with interest the study by Teng and colleagues reporting a high frequency $(11.4 \%)$ of epidermal growth factor receptor $(E G F R)$-activating mutations in triplenegative breast cancer (TNBC) in a Singapore cohort [1]. In a separate study, EGFR-activating mutations were detected in $7.7 \%$ ( 1 out of 13) of Chinese basal-like breast cancers [2]. Interestingly, this frequency ranged from 0 to $3 \%$ of other Asian and Caucasian patients [3, 4]. These results indicate that the frequency of these mutations may vary according to geographic and ethnic differences, as reported in non-small cell lung cancer, and these mutations appear to be limited mostly to Chinese patients with TNBC. In this study, in-depth characterization of these mutations in Chinese TNBC was attempted on retrospective archival tissues.

Fifty freshly frozen specimens of TNBC from patients without neoadjuvant chemotherapy were randomly selected from Zhejiang Cancer Hospital, China, from 2010 to 2011. The specimens were confirmed to be estrogen receptor- and progesterone receptor-negative, and less than $1 \%$ of tumor cells showed positive nuclear staining by an immunohistochemistry (IHC) assay. HER-2 negativity was defined as a score of 0 or $1+$ by IHC assay or a HER-2/chromosome 17 ratio of less than 2 and fewer than $4 H E R-2$ copies per nucleus by a fluorescence in situ hybridization assay. Written consent was obtained from all participants. This study was approved by the Research and Ethics Committee of Zhejiang Cancer Hospital.

Twenty-five EGFR-activating mutations (G719S, G719A, G719C, S768I, L858R, and L861Q and 19 mutations of

\footnotetext{
* Correspondence: wxiaojia@yahoo.com

'Department of Medical Oncology, Zhejiang Cancer Hospital, 38 Guangji Road, Hangzhou 310022, China

${ }^{2}$ Zhejiang Key Laboratory of the Diagnosis \& Treatment Technology on Thoracic Oncology, Zhejiang Cancer Hospital, 38 Guangji Road, Hangzhou 310022, China

Full list of author information is available at the end of the article
}

exon 19-Del) were analyzed by an Amplification Refractory Mutation System (ARMS) assay by using an ADx EGFR29 Mutation Kit (Amoy Diagnostics, Xiamen, China). The ARMS assay is able to detect mutations with allele frequencies as low as $1 \%$ [5]. Exons 18, 19, 20, and 21 were amplified by polymerase chain reaction assay, and all fragments were bidirectionally sequenced to screen for other mutations.

None of the EGFR-activating mutations was found by these two assays, but four single-nucleotide polymorphisms (SNPs) were identified by the sequencing assay (Table 1).

In silico prediction was performed to investigate the effect of the SNPs on gene splicing by using two programs: NNSplice 0.9 version (http://www.fruitfly.org/ seq_tools/splice.html) and Human Splicing Finder (http://www.umd.be/HSF3/). All four SNPs, particularly the c.2457G > A SNP, were predicted to alter splicing by one or both programs.

In summary, none of the well-known EGFR-activating mutations was identified in our cohort. This suggests that TNBCs form a group of cancers with marked heterogeneity. Targetable mutations may be present and clinically helpful in only a limited number of Chinese patients with TNBC.

\section{Abbreviations \\ ARMS: Amplification Refractory Mutation System; EGFR: Epidermal growth factor receptor; IHC: Immunohistochemistry; SNP: Single-nucleotide polymorphism; TNBC: Triple-negative breast cancer.}

\section{Competing interests}

The authors declare that they have no competing interests.

\section{Authors' contributions \\ WMC designed the study, performed in silico prediction of SNPs, and drafted the manuscript. YG prepared specimens for mutation analysis, collected the clinical data, and analyzed the sequencing data. XJW conceived and designed the study and revised the manuscript. All authors read and approved the final manuscript.}

\section{Biomed Central}


Table 1 Polymorphisms in exons 18, 19, 20, and 21 of EGFR in 50 triple-negative breast cancers

\begin{tabular}{|c|c|c|c|c|c|c|c|}
\hline \multirow[t]{2}{*}{ Exon } & \multirow[t]{2}{*}{ Position } & \multirow{2}{*}{$\begin{array}{l}\text { Amino acid } \\
\text { change }\end{array}$} & \multirow{2}{*}{$\begin{array}{l}\text { Minor allele } \\
\text { frequency } \\
\text { (number) }\end{array}$} & \multirow[t]{2}{*}{$\mathrm{dbSNP}$} & \multicolumn{2}{|l|}{ NNSplice } & \multirow[t]{2}{*}{ Human Splicing Finder } \\
\hline & & & & & $\begin{array}{l}\text { Score ratio of donor site } \\
\text { (SNP versus normal) }\end{array}$ & $\begin{array}{l}\text { Score ratio of } \\
\text { acceptor site } \\
\text { (SNP versus normal) }\end{array}$ & \\
\hline Intron 19 & c. $2283+103 C>T$ & - & $0.1(10)$ & rs17290371 & $0.48: 0.53$ & - & Creation of an intronic ESE site \\
\hline 20 & c. $2361 \mathrm{G}>\mathrm{A}$ & Q787Q & $0.18(9)$ & rs1050171 & $0: 0.43$ & - & Probably no impact on splicing \\
\hline \multirow[t]{2}{*}{20} & c. $2457 G>A$ & V819V & $0.04(1)$ & rs56183713 & $0.53: 0$ & 0.89:0.65 & 1. Creation of an exonic ESS site \\
\hline & & & & & & & 2. Alteration of an exonic ESE site \\
\hline Intron 20 & c. $2470-68 C>A$ & - & $0.02(1)$ & rs530416576 & - & - & Creation of an intronic ESE site \\
\hline
\end{tabular}

EGFR epidermal growth factor receptor, SNP single-nucleotide polymorphism, ESE exonic splicing enhancer, ESS exonic splicing silencer

\section{Authors' information}

XJW is the director of the Department of Breast Medical Oncology and the Department of Science and Education at Zhejiang Cancer Hospital in Hangzhou, China. His research interests include breast cancer, translational medicine, and breast cancer genetics. XJW has served as the principal investigator of several phase III breast cancer trials, such as those for Afinitor, Pertuzumab, and Lapatinib. XJW serves as a Chinese Anti-cancer Society Breast Cancer committee member and chairman designate of the Zhejiang Province Anti-cancer Society Breast Cancer Committee.

\section{Acknowledgments}

This study was supported by grants from the Science and Technology Program offered by the Health Bureau of Zhejiang Province, China (grants 2012RCB006 and 2014KYA006) and the Zhejiang Province Traditional Medical Science Fund Project of China (grant 2012ZB019). ARMS assay and sequencing were performed by the Innovation Center China, AstraZeneca Global R\&D, Shanghai, China.

\section{Author details}

${ }^{1}$ Department of Medical Oncology, Zhejiang Cancer Hospital, 38 Guangji Road, Hangzhou 310022, China. 'Zhejiang Key Laboratory of the Diagnosis \& Treatment Technology on Thoracic Oncology, Zhejiang Cancer Hospital, 38 Guangji Road, Hangzhou 310022, China. ${ }^{3}$ Institute of Cancer Research, Zhejiang Cancer Hospital, 38 Guangji Road, Hangzhou 310022, China.

\section{Published online: 20 August 2015}

\section{References}

1. Teng YH, Tan WJ, Thike AA, Cheok PY, Tse GM, Wong NS, et al. Mutations in the epidermal growth factor receptor (EGFR) gene in triple negative breast cancer: possible implications for targeted therapy. Breast Cancer Res. 2011;13:R35

2. Lv N, Lin S, Xie Z, Tang J, Ge Q, Wu M, et al. Absence of evidence for epidermal growth factor receptor and human homolog of the Kirsten rat sarcoma-2 virus oncogene mutations in breast cancer. Cancer Epidemiol. 2012:36:341-6.

3. Park HS, Jang MH, Kim EJ, Kim HJ, Lee HJ, Kim YJ, et al. High EGFR gene copy number predicts poor outcome in triple-negative breast cancer. Mod Pathol. 2014;27:1212-22.

4. Jacot W, Lopez-Crapez E, Thezenas S, Senal R, Fina F, Bibeau F, et al. Lack of EGFR-activating mutations in European patients with triple-negative breast cancer could emphasise geographic and ethnic variations in breast cancer mutation profiles. Breast Cancer Res. 2011;13:R133.

5. Bai H, Wang Z, Chen K, Zhao J, Lee JJ, Wang S, et al. Influence of chemotherapy on EGFR mutation status among patients with non-small-cell lung cancer. J Clin Oncol. 2012;30:3077-83. 